\title{
The forgotten numbers: A closer look at COVID-19 non-fatal valuations
}

\author{
Thomas J. Kniesner ${ }^{1} \cdot$ Ryan Sullivan ${ }^{2}$
}

Accepted: 21 October 2020 / Published online: 4 November 2020

(C) This is a U.S. government work and not under copyright protection in the U.S.; foreign copyright protection may apply 2020

\begin{abstract}
Our research estimates COVID-19 non-fatal economic losses in the U.S. using detailed data on cumulative cases and hospitalizations from January 22, 2020 to July 27, 2020, from the Centers for Disease Control and Prevention (CDC). As of July 27, 2020, the cumulative confirmed number of cases was about 4.2 million with almost 300,000 of them entailing hospitalizations. Due to data collection limitations the confirmed totals reported by the $\mathrm{CDC}$ undercount the actual number of cases and hospitalizations in the U.S. Using standard assumptions provided by the CDC, we estimate that as of July 27 , 2020, the actual number of cumulative COVID-19 cases in the U.S. is about 47 million with almost 1 million involving hospitalizations. Applying value per statistical life (VSL) and relative severity/injury estimates from the Department of Transportation (DOT), we estimate an overall non-fatal unadjusted valuation of $\$ 2.2$ trillion for the U.S. with a weighted average value of about $\$ 46,000$ per case. This is almost $40 \%$ higher than the total valuation of $\$ 1.6$ trillion (using about $\$ 11$ million VSL from the DOT) for all approximately 147,000 COVID-19 fatalities. We also show a variety of estimates that adjust the non-fatal valuations by the dreaded and uncertainty aspect of COVID-19, age, income, and a factor related to fatality categorization. The adjustments show current overall non-fatal valuations ranging from about $\$ 1.5$ trillion to about $\$ 9.6$ trillion. Finally, we use CDC forecast data to estimate non-fatal valuations through November 2020, and find that the overall cumulative valuation increases from about $\$ 2.2$ trillion to about $\$ 5.7$ trillion or to about $30 \%$ of GDP. Because of the larger numbers of cases involved our calculations imply that non-fatal infections are as economically serious in the aggregate as ultimately fatal infections.
\end{abstract}

Keywords Value ofa Statistical Injury · VSI · Value of a Statistical Life - VSL · COVID-19. Pandemic $\cdot$ Willingness to Pay · Benefit-Cost Analysis · CDC

Thomas J. Kniesner

tom.kniesner@cgu.edu

1 Claremont Graduate University, Syracuse University (Emeritus), IZA, Claremont, CA, USA

2 Naval Postgraduate School, Monterey, CA, USA 
JEL Classification $\mathrm{I} 18 \cdot \mathrm{J} 17 \cdot \mathrm{J} 28 \cdot \mathrm{H} 51$

"... this is not the end. It is not even the beginning of the end, but it is, perhaps, the end of the beginning."

Sir Winston Churchill, November 10, 1942

\section{Introduction}

The Sir Winston Churchill quote above was about the first Allied victory in World War II in North Africa but is apropos to the current fight against the ongoing pandemic in the United States. Just as Churchill hoped that the end was beginning we too hope the end may be starting. The contention that the current pandemic is as much of an economic as a medical problem has been argued eloquently in many places in the economics and more popular publications (Interlandi 2020; Manski 2020a, 2020b). Although not the only economists to mention the underemphasized non-fatal dimensions of COVID-19 infections (see Viscusi (2020) in this issue), we demonstrate that policy discussions have underemphasized the importance of addressing the spread of COVID-19 for patients who survive when calculating the net benefits of limiting social contact and the resulting negative economic consequences.

In the model research to date on the net economics benefits of intervening in the spread of the COVID-19 pandemic the net benefit is $N B=V S L \times\left(D_{1}-D_{2}\right)$ $-\sum_{t=0}^{T}\left(Y_{1 t}-Y_{2 t}\right)(1+r)^{-t}$, which is the value of lives saved by social distancing minus the present value of GDP lost due to social distancing, which depend on the number of deaths with and without social distancing and the present value of the two alternate GDP streams. There is much more to the story, however. The list of the consequences of non-fatal cases seems to be extended regularly with alarming implications. Dr. Henry J. Miller, founding director of the FDA's Office of Biotechnology, emphasizes that COVID-19 is "more than a transient, self-limited respiratory infection." He notes the list of reported problems includes many non-respiratory complications such as loss of smell or taste, confusion and cognitive impairments, fainting, sudden muscle weakness or paralysis, seizures, ischemic strokes, kidney damage, abnormal bloodcoagulation tests, transmission to an unborn child via the placenta, and a severe (though rare) pediatric inflammatory syndrome. Additionally important is the dread factor that COVID-19 creates. Dr. Miller emphasizes that the disease is much different than the previous flu diseases where recovery can be accompanied with more permanent adverse outcomes such as chronic fatigue syndrome, whose list of symptoms include loss of memory or concentration and tiredness that can lead to complete debilitation that can persist for years. The policy implication is that we need to suppress and mitigate COVID-19 not just for the reduction of current medical care costs and prevention of fatalities.

In what follows in Section 2 we demonstrate that the number of non-fatal COVID19 cases is possibly much more sizeable than what is typically in the discussion of the pandemic and how widespread it is. In Section 3 we then use multiple comprehensive measures of willingness to pay to avoid non-fatal health losses to monetize the economic losses from COVID-19 that have been largely ignored in the literature which 
emphasizes fatalities. The point is not to downplay fatalities but rather emphasize a largely ignored component of the costs of the pandemic that when added up give additional importance to the value of reduced contact via wearing masks and socially distancing. Section 4 discusses the likely path the pandemic will take in the near future and its consequences for the social calculus of limiting the infection's spread in the absence of a vaccine. Section 5 concludes with a reiteration of the future research and policy implications of our data that the benefits of interdicting the spread of COVID-19 may be twice as high as typically used in comparing the benefits of slowing economic activity versus the cost.

\section{Current situation}

\subsection{CDC confirmed cases and hospitalizations}

The CDC provides daily updates on confirmed COVID-19 cumulative case totals for all states (CDC 2020a). The first confirmed case according to the CDC was recorded in Washington State on January 22, 2020. The most recently available data show a cumulative total of about 4.2 million confirmed COVID-19 cases in the United States between January 22, 2020, through July 27, 2020.

Figure 1 shows the distribution of cumulative COVID-19 cases across different states as of July 27, 2020. California has the highest cumulative total with a reported

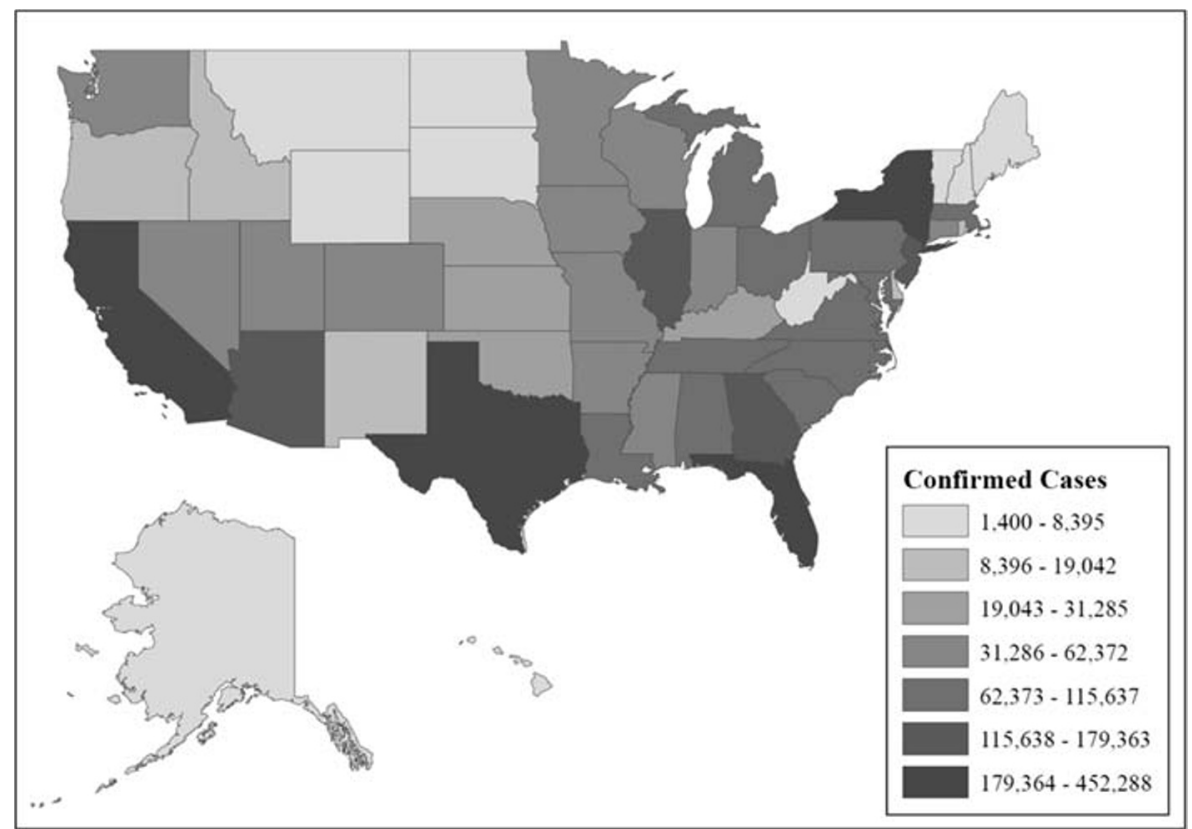

Fig. 1 CDC Confirmed COVID-19 Cases by State as of July 27, 2020. Notes: Data taken from CDC ( 2020a) as viewed on July 27, 2020. Available at: https://github.com/CSSEGISandData/COVID-19/tree/master/csse_ COVID_19_data/csse_COVID_19_daily_reports_us 
452,000 cases. Florida $(423,000)$, New York $(412,000)$, Texas $(394,000)$, and New Jersey $(179,000)$ round out the top five highest number of cases by state. In contrast, Vermont (1400), Hawaii (1700), Wyoming (2500), Alaska (2500), and Montana (3300) round out the bottom five in terms of lowest number of confirmed cases by state.

The confirmed case totals as documented by the CDC are known to be a substantial undercount in comparison to the actual number of infections in the United States. The undercount is because there were few testing sites available early on in the pandemic and even today people often do not get tested for a variety of reasons (no or few symptoms, testing availability). On June 25, 2020, CDC Director Robert Redfield highlighted the case measurement accuracy issue with the confirmed case totals and stated that "our best estimate right now is for every case reported there were actually 10 other infections." At the time of Director Redfield's statement there were roughly 2.4 million confirmed cases, so media outlets reported that the actual number of cases in the United States was "at least 24 million" (New York Times 2020; Washington Post 2020).

In terms of confirmed cumulative hospitalization totals the CDC reports a total of about 293,000 confirmed hospitalizations from January 22, 2020, through July 27, 2020 (CDC 2020a). The CDC hospitalization data is limited in a variety of ways. For example, only 36 states report cumulative hospitalization numbers in the CDC dataset. ${ }^{1}$ In addition, most states did not track cumulative hospitalization numbers until the pandemic was well under way so the confirmed numbers provided here are likely substantially undercounted. Finally, breakdowns in types of hospitalization are even more limited with many of the states not reporting any information on categories such as ICU and mechanical ventilator rates for patients.

Figure 2 shows confirmed cumulative COVID-19 hospitalization totals by state from January 22, 2020, through July 27, 2020. New York has by far the highest number of hospitalizations with a total of about 90,000. Florida $(24,000)$, New Jersey $(21,000)$, Georgia $(17,000)$, and Maryland $(12,000)$ round out the top five highest number of hospitalizations by state. That said, some of the states with the highest number of cases as shown in Fig. 1 do not provide cumulative hospitalization totals such as California, Texas, and Illinois. As indicated earlier, the hospitalization totals shown here are undercounts of the true numbers in the United States.

\subsection{Current non-fatal cases by severity}

Panel A in Table 1 shows the current CDC confirmed cumulative totals from January 22, 2020, through July 27, 2020, for cases (4.2 million), deaths (about 147,000), recovered cases (1.3 million), active cases (2.8 million), and hospitalizations (about

\footnotetext{
${ }^{1}$ Almost all of the states are starting to track at least some totals on the number of hospitalizations. For example, two of the largest states (California and Texas) have some limited information about hospitalizations on their state websites. The CDC still does not provide specific cumulative totals for California or Texas at the time of our analysis, however.
} 


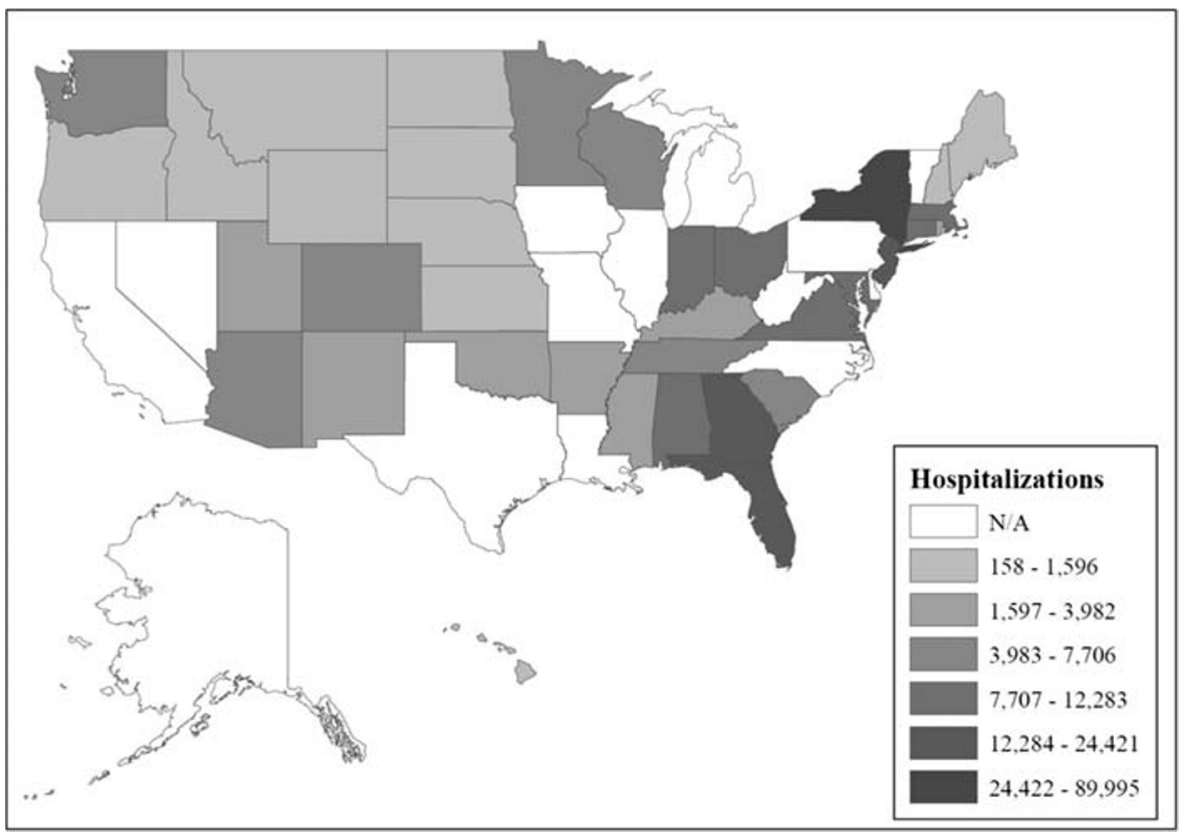

Fig. 2 CDC Confirmed COVID-19 Hospitalizations by State as of July 27, 2020. Notes: Data taken from CDC ( 2020a) as viewed on July 27, 2020. Available at: https:/github.com/CSSEGISandData/COVID-19/ tree/master/csse_COVID_19_data/csse_COVID_19_daily_reports_us

293,000). ${ }^{2}$ These are the five primary outcomes available in the most up-to-date CDC data (CDC 2020a).

Given the limited documentation by many of the states on the type of hospitalization (ICU rates and percentage of patients on mechanical ventilator) and case type (asymptomatic versus symptomatic) the CDC provides best case estimates in their Planning Scenarios file (CDC 2020b) for a variety of categories. The estimates/assumptions are shown in Panel B in Table 1. The best estimates from the CDC are that (a) $40 \%$ of cases are asymptomatic (b) $32 \%$ of hospitalizations are categorized as ICU patients (c) $75 \%$ of ICU patients are put on mechanical ventilation and (d) about $3 \%$ of symptomatic cases end up in the hospital.

Panel A in Table 2 shows the current breakdown of the categories we use for the non-fatal results using total confirmed cases (4.2 million) and hospitalizations $(293,000)$ from the CDC from January 22, 2020, through July 27, 2020 (CDC 2020a). We use the assumptions provided by the CDC as described in Table 1 (CDC 2020b; CDC 2020c) as a basis to provide totals on the number of asymptomatic cases (1.7 million), symptomatic cases with no hospitalization (2.2 million), hospitalization totals minus those in ICU and on ventilators $(199,000)$, ICU total minus those on ventilators $(23,000)$, and ICU total on mechanical ventilation $(70,000)$.

\footnotetext{
${ }^{2}$ It is possible that the confirmed number of COVID-19 deaths in the CDC data undercounts the actual values. For example, Weinberger et al. (2020) find the number of excess deaths due to any cause (in comparison to previous years) increased by approximately 122,000 from March 1, 2020, to May 30, 2020. As a reference point, this is $28 \%$ higher than the reported number of COVID-19 deaths during that same time period.
} 


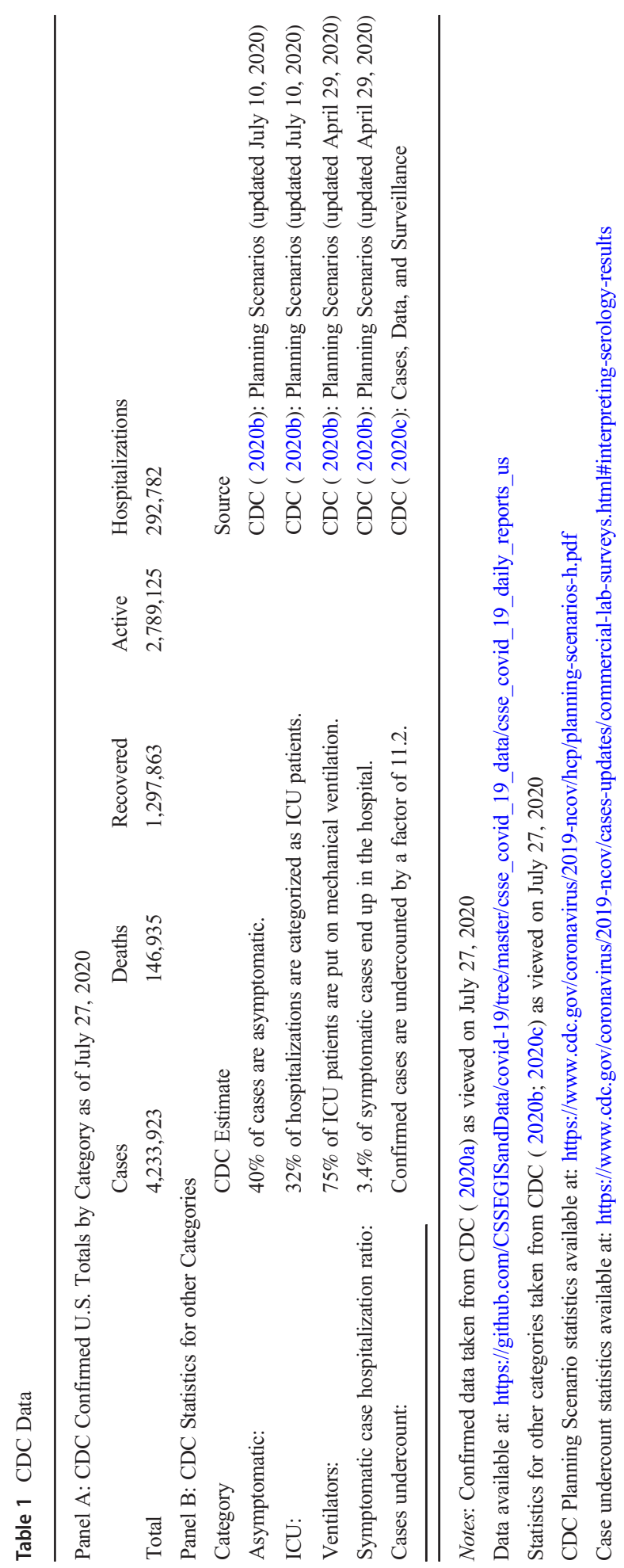


Table 2 Current Non-Fatal Valuations as of July 27, 2020

\begin{tabular}{|c|c|c|c|}
\hline & Total & Severity Category & Overall Valuation (in billions) \\
\hline \multicolumn{4}{|c|}{ Panel A: Current Number of Confirmed Non-Fatal Totals and Valuations as of July 27, 2020} \\
\hline Asymptomatic cases (no hospitalization) & $1,693,569$ & $\$ 10,900$ & $\$ 18.5$ \\
\hline Symptomatic cases (no hospitalization) & $2,247,572$ & $\$ 32,700$ (Minor) & $\$ 73.5$ \\
\hline \multicolumn{4}{|l|}{ Hospitalization total (minus those } \\
\hline in ICU and on ventilators) & 199,092 & $\begin{array}{l}\$ 512,300 \\
\quad \text { (Moderate) }\end{array}$ & $\$ 102.0$ \\
\hline \multicolumn{4}{|l|}{ ICU total (minus } \\
\hline those on ventilators) & 23,423 & $\$ 1,144,500$ (Serious) & $\$ 26.8$ \\
\hline \multicolumn{4}{|l|}{ ICU total } \\
\hline on mechanical ventilation & 70,268 & $\$ 2,899,400$ (Severe) & $\$ 203.7$ \\
\hline Total cases and overall valuation & $4,233,923$ & & $\$ 424.5$ \\
\hline \multicolumn{4}{|l|}{ Non-Fatal Weighted Average Value } \\
\hline \multicolumn{2}{|l|}{ (Overall valuation/Total number of cases) } & \multicolumn{2}{|l|}{$\$ 100,260$} \\
\hline \multicolumn{4}{|c|}{ Panel B: Estimated Current Number of Actual Non-Fatal Totals and Valuations as of July 27, 2020} \\
\hline Asymptomatic cases (no hospitalization) & $18,967,975$ & $\$ 10,900$ & $\$ 206.8$ \\
\hline Symptomatic cases (no hospitalization) & $27,484,596$ & $\$ 32,700$ (Minor) & $\$ 898.7$ \\
\hline \multicolumn{4}{|l|}{ Hospitalization total (minus those } \\
\hline in ICU and on ventilators) & 657,809 & $\begin{array}{l}\$ 512,300 \\
\quad \text { (Moderate) }\end{array}$ & $\$ 337.0$ \\
\hline \multicolumn{4}{|l|}{ ICU total (minus } \\
\hline those on ventilators) & 77,389 & $\$ 1,144,500$ (Serious) & $\$ 88.6$ \\
\hline \multicolumn{4}{|l|}{ ICU total } \\
\hline on mechanical ventilation & 232,168 & $\$ 2,899,400$ (Severe) & $\$ 673.1$ \\
\hline Total cases and overall valuation & $47,419,938$ & & $\$ 2204.2$ \\
\hline \multicolumn{4}{|l|}{ Non-Fatal Weighted Average Value } \\
\hline (Overall valuation/Total number of cases) & & $\$ 46,483$ & \\
\hline
\end{tabular}

Notes: Confirmed data taken from CDC ( 2020a) as viewed on July 27, 2020

Data available at: https://github.com/CSSEGISandData/Covid-19/tree/master/csse_covid_19_data/csse_ covid_19_daily_reports_us

Assumptions for Panel B taken from CDC ( 2020b; 2020c) as detailed in Table 1

As discussed above the number of CDC confirmed cases substantially understates the actual number of infections in the United States. Due to the limitation with the confirmed case data the CDC decided to partner with commercial laboratories to conduct a large-scale geographic seroprevalence survey to better understand the magnitude of the case undercounts in the United States (CDC 2020c). This was the same survey data that CDC Director Redfield cited for why he viewed the confirmed case data as being under-representative of the actual counts in society.

The surveys were conducted in six different locations across the United States from March 23, 2020 through May 3, 2020 (CDC 2020c). Samples were taken from blood specimens tested for a variety of reasons unrelated to COVID-19 to identify infection rates and compare them with reported rates by the $\mathrm{CDC}$ in the same geographic region. 
The survey results indicated that infection rates were under-reported by a factor of 6-24 times the reported confirmed case totals by the CDC. For our purposes here we use the median value in the survey, which states that infection rates are under-reported by a factor of about 11. This is shown in the bottom part of Panel B in Table 1 and matches up relatively closely to the value CDC Director Redfield cited (10 additional infections for every one confirmed case) in his media correspondence.

Panel B in Table 2 shows the results when the estimated number of actual cases are in the calculation instead of confirmed cases. To find the total number of estimated actual cases between January 22, 2020, and July 27, 2020, we use the total number of confirmed cases (4.2 million) and multiply it by the adjustment factor of 11 as detailed by the CDC (CDC 2020a; CDC 2020c). The cumulative number of actual cases during January-July 2020 is about 47.4 million. Breakdowns by non-fatal category can then be calculated by using the assumptions as detailed in Panel B in Table 1. Using the information just mentioned we estimate totals for actual number of asymptomatic cases (19.0 million), symptomatic cases with no hospitalization (27.5 million), hospitalization total minus those in ICU and on ventilators $(658,000)$, ICU total minus those on ventilators $(77,000)$, and ICU total on mechanical ventilation $(232,000)$.

\section{Economic losses from non-fatal cases}

\subsection{Valuing non-fatal cases using fractions of VSL lost}

We first use the Department of Transportation (2016) guidance on value per statistical life (VSL) and severity/injury estimates as a basis for our non-fatal valuations by category. After updating the figures for earnings and inflation the DOT guidance recommends using a VSL of about \$11 million in 2019 dollars. We use the severity classifications in the DOT guidance as a basis for our non-fatal valuations. DOT (2016) recommends using six different severity categories in benefit-cost analyses including Level 1 (minor), which corresponds to using a $0.3 \%$ amount of the VSL, Level 2 (moderate), which uses about a 5\% amount, Level 3 (serious), which uses about a 10\% amount, Level 4 (severe), which uses about a 27\% amount fraction, Level 5 (critical), which uses about a 59\% amount, and Level 6 (unsurvivable) which uses a $100 \%$ amount (the full VSL).

We therefore value asymptomatic cases at about $\$ 11,000$ (in 2019 dollars) each of which corresponds to using a $0.1 \%$ amount of the VSL in DOT (2016). ${ }^{3}$ Symptomatic cases with no hospitalizations are assumed to line up in the minor category (about $\$ 33,000$ each). Hospitalizations not in ICU or on a ventilator are classified in the moderate category $(\$ 512,000$ each). ICUs without being on a ventilator are classified in the serious category ( $\$ 1.1$ million each). ICUs on a mechanical ventilator are classified in the severe category (\$2.9 million each). We view such

\footnotetext{
${ }_{3}^{3}$ Given the relatively minor impact of the asymptomatic cases we were unable to directly match that grouping to any of the standard DOT severity classifications. The evidence to date suggest possibly up to half of the asymptomatic cases may have some minor lung damage even though the infected individuals do not realize it (Oran and Topol 2020). Given the uncertainty of the virus and its long-term health outcomes, possible isolation effects, and other factors such as dread, we do not believe a value of $\$ 0$ for asymptomatic cases is appropriate, and we examine the issue of economic losses for asymptomatic cases further in Section 3.5.
} 
calculations as providing something approaching an upper bound, although we will argue below that non-fatal COVID-19 losses could be adjusted upward from the DOT categorical losses not only because of the uncertainty we mentioned earlier that happens over the course of possible non-fatal complications but also because of the so-called dread factor, which we elaborate on below.

Before proceeding it is important to note that the DOT (2016) injury categories we apply now are directly based on research by Spicer and Miller (2010). They compute injury utility weights from six different health and quality of life dimensions (mobility, cognitive, activities of daily living, pain, sensory, and cosmetic aspects of functioning). The original utility weights come from subjective analysis of four physicians with expertise in orthopedics, neurology, surgery and plastic surgery. The utility weights were then converted into quality-adjusted life year (QALY) estimates using a review of the academic literature.

Although the original utility weights are subjective the transition to QALY and fractional VSL estimates have their roots in the willingness-to-pay literature, which is more widely accepted in the field of economics. Spicer and Miller (2010) estimate minor injuries as 0.06 QALY lost, moderate injuries as 0.85 QALY lost, serious injuries as 1.77 QALY lost, severe injuries as 4.9 QALY lost, and critical injuries as 11.1 QALY lost (see Table 10 in their original paper for details). Their QALY values were then taken by DOT (2016) and converted into VSL fractions, which we use here.

A limitation of the DOT (2016) estimates is that they are specific for injuries and primarily relatable to vehicle accidents. In contrast, here we are trying to map in COVID-19 non-fatal valuations based off of health status. Due to the uncertain longterm health effects of COVID-19, it is possible that categories do not match up exactly. For example, some of the more severe cases of COVID-19 could take away far more QALYs than the severe status indicates (and could be categorized as critical or even worse). In contrast, it is possible that some of the asymptomatic cases could simply be categorized as having lost zero QALYs.

We relied on our professional judgement for the mapping system used here and certainly recognize the highly uncertain nature of using the DOT (2016) values. Some of the values could be lower or higher, but we believe that on average the estimates we use across the categories should be relatively close to their true values. However, it is critical for researchers to provide a range of values when doing sensitivity checking as noted in Section 3.7.

Panel A in Table 2 multiplies the confirmed totals for the different non-fatal classifications by their respective DOT severity values. The overall valuation for all confirmed cases and hospitalizations equals about $\$ 424$ billion as of July 27, 2020. The weighted average COVID-19 non-fatal value is the overall valuation ( $\$ 424$ billion) divided by the total number of cases (4.2 million). The confirmed COVID-19 non-fatal weighted average value from January 22, 2020, through July 27, 2020, is about $\$ 100,000$.

To obtain the estimated overall valuations in Panel B in Table 2, we multiply the non-fatal category totals by their respective DOT severity values. We sum the values for all non-fatal categories including asymptomatic cases (\$207 billion), symptomatic cases with no hospitalization (\$899 billion), hospitalizations not in ICU or on a ventilator ( $\$ 337$ billion), ICUs without being on a ventilator ( $\$ 89$ billion), and ICUs on a mechanical ventilator ( $\$ 673$ billion) to obtain an overall non-fatal valuation of 
about \$2.2 trillion. As a comparison the overall fatality valuation total as of July 27 , 2020 , is $\$ 1.6$ trillion ( 147,000 deaths times the $\$ 11$ million VSL from the DOT).

The overall valuation total ( $\$ 2.2$ trillion) divided by the total number of cases $(47.4$ million) provides a COVID-19 non-fatal weighted average value of about $\$ 46,000$. As a judge of its reasonableness we note that other valuations we develop below are surprisingly similar (with the possible exception of adjusting the valuation by the dread and uncertainty factors).

\subsection{Age adjustment}

There have been a number of papers in the growing body of COVID-19 research that discuss how adjusting the VSL by various personal characteristics can dramatically impact the overall valuation of COVID-19 fatalities (Adler 2020; Greenstone and Nigam 2020; Hall et al. 2020; Hammitt 2020; Robinson et al. 2020; Thunström et al. 2020; Viscusi 2020). The most common examples include adjusting the average population-wide VSL by age, income, or the dreaded and uncertainty aspects of the disease. The non-fatal valuations presented here face similar issues and adjusting the values by some of these factors could change the results, possibly by a substantial amount. For example, the non-fatal values which we calculate are all based upon some fraction of the average population-wide VSL as directed by DOT (2016). If the COVID-19 specific VSL is different than the average population-wide VSL, then the non-fatal values could also be adjusted for similar reasons.

To date, the most common modification of the COVID-19 VSL has been to adjust the value by age. Greenstone and Nigam (2020) is an early study to do this and their main results use an average COVID-19 VSL of $\$ 4.5$ million in comparison to an assumed U.S. Government VSL of $\$ 11.5$ million per statistical life. The Greenstone and Nigam (2020) results translate into about a 60\% discount for the COVID-19 VSL in comparison to the average population-wide estimate. Robinson et al. (2020) estimate an age-adjusted COVID-19 VSL using a constant value per statistical life-year (VSLY) approach and another approach that follows an inverse-U shaped pattern. They find the VSLY approach discounts the COVID-19 VSL by about $60 \%$ in comparison to the average population wide estimate, and the inverse- $\mathrm{U}$ method discounts it by about $20 \%$. Viscusi (2020) uses a VSLY approach and finds the COVID-19 VSL is discounted by about $50 \%$. There appears to be a wide range of results in the literature on the age-VSL connection with a significant amount of uncertainty. Applying similar methods to nonfatal categories provides comparable uncertainties on the overall valuations.

Adjusting the COVID-19 non-fatal values by age is quite different in comparison to calculating the values for statistical fatalities. A main reason is that the distribution for COVID-19 cases follows a much different age pattern than in the fatality data. Previous work by the CDC (2020e) on the demographics of COVID-19 victims with tabulations for cases, hospitalizations, and deaths has shown fatalities are much more skewed toward the elderly than some of the other categories, particularly in comparison to the case totals (see Fig. 3 for details). For example, the number of cases is largely concentrated among the middle-aged portion of the U.S. population with $65 \%$ of the cases confirmed among the 20-59 age group (CDC 2020e). This is in stark contrast with the fatality data which shows $81 \%$ of the fatalities occurring among those aged 65 and above (Robinson et al. 2020). Hospitalizations are skewed more heavily toward the 
a Cases by Age Group

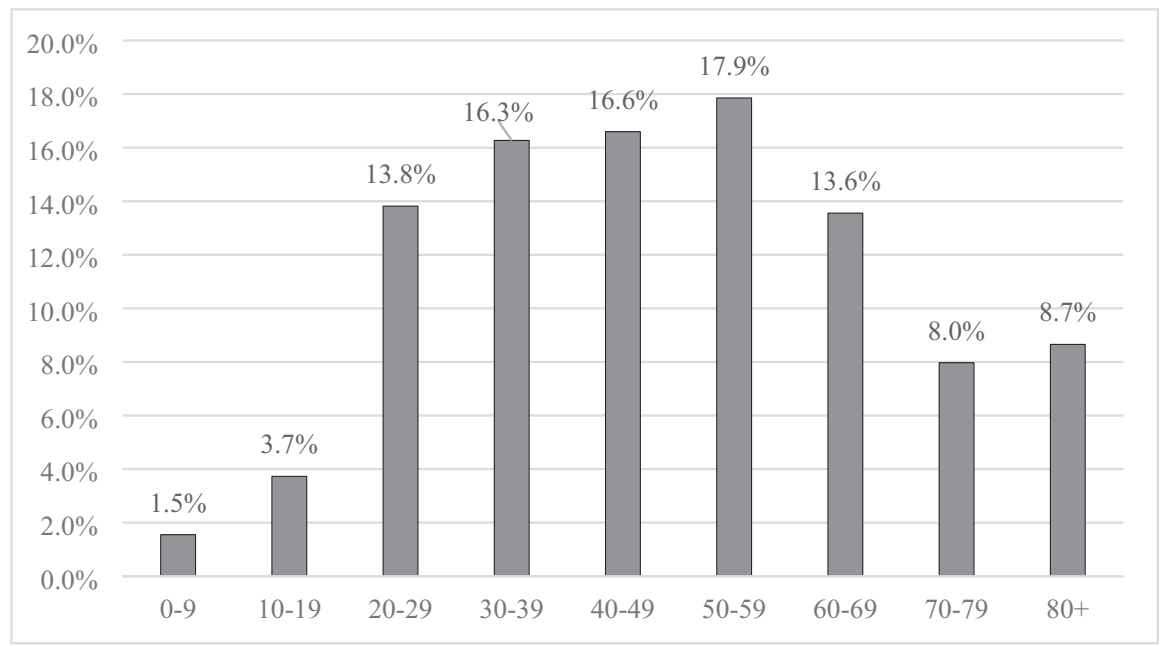

\section{b Hospitalizations by Age Group}

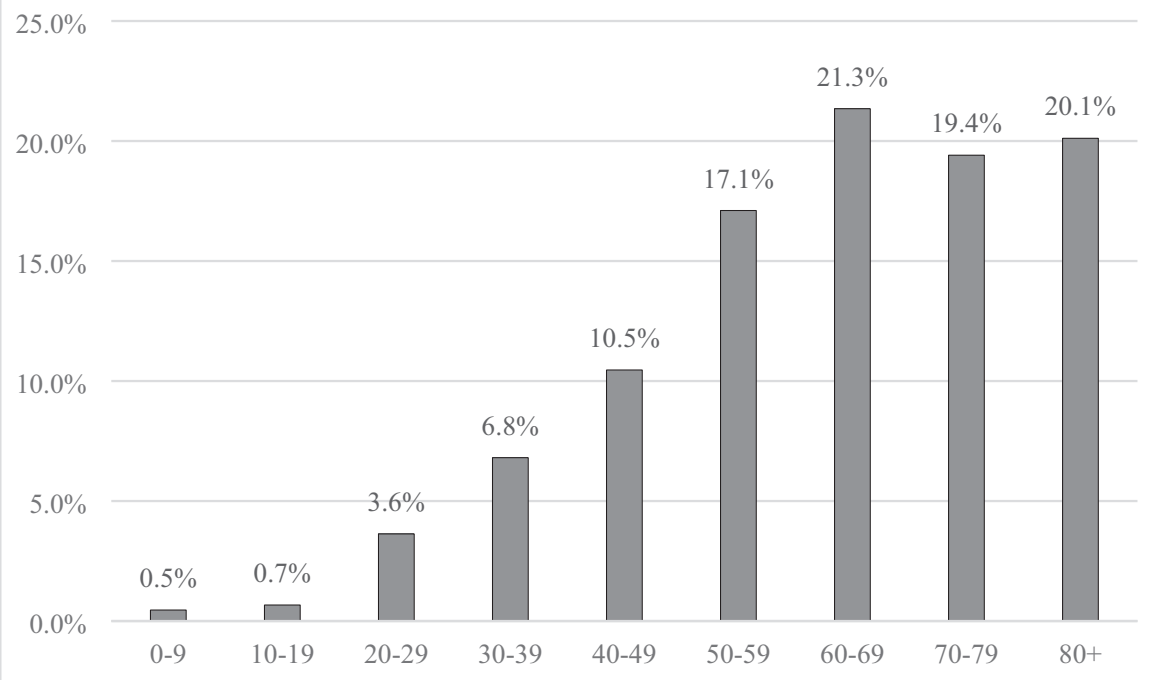

Fig. 3 Age Distribution for Laboratory Confirmed Cases and Hospitalizations. Panel A: Cases by Age Group, Panel B: Hospitalizations by Age Group. Notes: Data taken from Coronavirus Disease 2019 Case Surveillance. United States, January 22-May 30, 2020. CDC Morbidity and Mortality Weekly Report. Available at: https://www.cdc.gov/mmwr/volumes/69/wr/pdfs/mm6924e2-H.pdf

elderly in comparison to the number of cases. The CDC ( 2020e) data show that about $60 \%$ of the hospitalizations take place among the $60+$ age group. In short, it is possible that adjusting the non-fatal valuations by age will bring down the values, but it would be a much smaller adjustment in comparison to the adjusted values in the regular COVID-19 VSL literature. 
As a sensitivity analysis, we apply the age adjustment ratios (60\% discount for the VSLY method and $20 \%$ for the inverse-U method) in Robinson et al. (2020) to the hospitalization values in Panel B in Table 2. While we adjust the hospitalization values for age, we assume the asymptomatic case values and symptomatic case values with no hospitalization remain the same (because the categories are not skewed toward the elderly). We find overall valuations in Table 2 decrease from $\$ 2.2$ trillion to $\$ 1.6$ trillion (about a 30\% decrease) when using the VSLY method and fall to about $\$ 2$ trillion (about a 10\% decrease) when using the inverse-U method. Applying the age adjustment ratio from Greenstone and Nigam (2020) similarly decreases the overall non-fatal valuation from about $\$ 2.2$ trillion to about $\$ 1.5$ trillion (a 30\% decrease).

\subsection{Income adjustment}

Another factor that might alter the estimates would be applying a value adjustment based on wealth or income status. Recent data suggests COVID-19 has disproportionality impacted lower income class families in comparison to higher income families across the United States (CMS 2020). Lower incomes generally translate into lower willingness to pay values in relation to preventing fatal and nonfatal injuries.

Although the magnitude of the income effect varies significantly depending upon the data and method used the income elasticity literature has found a $10 \%$ decrease in income translates into roughly a 10\% decrease in the WTP estimates (Hammitt and Robinson 2011; Lindhjem et al. 2011; Kniesner et al. 2010; Viscusi and Aldy 2003; Viscusi and Masterman 2017a; Viscusi and Masterman 2017b; Masterman and Viscusi 2018). As a reference the guidance from the DOT and HHS recommends using an income elasticity equal to 1.0 for the types of valuations we do here, which closely follows the literature mentioned above (U.S. Department of Transportation 2016; U.S. Department of Health and Human Services 2016).

At the current time, data available on income levels for those impacted by COVID19 are limited. Thus, we do not provide sensitivity analysis adjustment values for income levels due to the unavailability of the needed income data, which might prove informative in the future. It is likely that the estimates provided in Table 2 would be lower but the magnitude is uncertain given the current data limitations.

\subsection{Dread and uncertainty over the outcome of the disease}

The dread and uncertainty factors of COVID-19 could also play significant roles in how the valuations might change in a sensitivity analysis. Previous literature has shown individuals place a premium on avoiding particularly dreaded disease types and those with significant uncertainty (Chilton et al. 2006; Hammitt and Liu 2004; Jones-Lee and Loomes 1995; Liu et al. 2005; McDonald et al. 2016; Riddel and Shaw 2006; Viscusi 2019; Miller 2020). The COVID-19 pandemic has similar features in regard to the dreaded and uncertainty aspects of the disease (uncertainty in the infection rates and long-term outcomes such as reduced lung-capacity). For emphasis we note a few paragraphs from a recent column written by well-known LA Times sportswriter Bill Plaschke where he describes his experience with getting COVID-19 and the dread it brings. 
"Nobody tells you about the dread. From the moment that my doctor phoned me with the test results, to the moment I am writing this column, I have been scared out of my mind. I know the minuscule overall fatality percentages. I know the overwhelming odds of survival for a 61-year-old male in good health with no preexisting conditions. It doesn't matter. Once you realize you have a virus that could kill you and there's nothing anybody can do about it, you live in constant fear. With every trickle of sweat off your forehead, you worry. With every deep cough, you wonder. You check your temperature 53 times every day, and every single time that thermometer is in your mouth, you close your eyes and pray. You stick your finger in the pulse oximeter every hour, and beg for the number to rise. ... And then there are the late nights. ... You can't call your doctor because he's already told you that there's nothing he can do. ... you simmer alone in the darkness doing nothing, paralyzed by fear and chasing your breath and praying that 102.1 does not become 103.1." (Plaschke 2020, boldface added).

Willingness to pay or accept estimates from the literature to avoid cancer is one possible approach to adjust the COVID-19 non-fatal values for the dreaded and uncertainty aspects of the disease. A wide range of estimates has emerged in the literature by evaluating unique situations such as avoiding cancer risks from nuclearwaste (Riddel and Shaw 2006), arsenic in drinking water (Viscusi et al. 2014), and superfund sites (Gayer et al. 2000) to name a few. The literature has found a cancer premium of around $20 \%$ in comparison to the normal population-wide VSL (Viscusi 2019). Applying a $20 \%$ premium on the non-fatal valuations in Panel B in Table 2 would raise the overall values from about $\$ 2.2$ trillion to about $\$ 2.6$ trillion.

Possibly the closest example we have in the literature on how to adjust the values for the dread and uncertainty aspect for COVID-19 comes from the estimates provided in Liu et al. (2005), who use contingent valuation methods to estimate WTP values to avoid the threat of the severe acute respiratory syndrome (SARS) epidemic in Taiwan in the early 2000s. They find VSL estimates ranging in value from about $\$ 3$ million to about $\$ 12$ million (U.S. dollars) in comparison to previous normal VSL estimates in the population of Taiwan which range from $\$ 360,000$ to $\$ 2.2$ million. The results suggest the dreaded and uncertainty aspects of SARS may increase the WTP values by roughly $25 \%$ to $435 \%$. Gyrd-Hansen et al. (2008) find a similar multiplier $(3=>200 \%)$ for a serious influenza pandemic in Norway in interview data. Applying the numbers to the COVID-19 non-fatal values in Panel B in Table 2 suggests that the original non-fatal overall valuation of $\$ 2.2$ trillion might be as high as $\$ 2.8$ trillion to $\$ 9.6$ trillion. ${ }^{4}$

\subsection{How deaths are categorized}

The penultimate factor we consider that could impact the analysis is how deaths are distributed among the various non-fatal categories in Table 2. As of July 27, 2020, there

\footnotetext{
${ }^{4}$ Finally, Rowell (2020) has recently suggested using EPA morbidity valuations from relatively similar respiratory diseases as another option to apply in sensitivity analyses. The EPA has previously used a $\$ 740,000$ value (in 2019 dollars) for cases of chronic bronchitis in benefit-cost analyses on air pollution (U.S. Environmental Protection Agency 2009). Assuming a \$740,000 valuation for all 28 million of the COVID-19 hospitalizations and symptomatic cases with no hospitalizations in Panel B in Table 2 results in an overall valuation of about $\$ 21$ trillion.
} 
have been a total of about 147,000 COVID-19 deaths in the CDC data. Panel B in Table 2 estimates that there was a total of 47.4 million cases from January 22, 2020 through July 27,2020 . Thus, there should technically be a total of 47.3 million nonfatal cases in the final calculations.

The CDC documentation on where deaths took place (at home, in the hospital) is still quite limited at this time and the distribution of cases across the various categories is highly speculative. ${ }^{5}$ For example, if all of the fatalities $(147,000)$ were on ICU mechanical ventilators at the time of death then they should be excluded in the overall non-fatal valuation totals (because they should be categorized as fatalities). On the other extreme, if the deaths took place suddenly, before requiring hospitalization of the individuals, then the values would have to be taken out of the asymptomatic or symptomatic cases with no hospitalization totals.

We provide a range of estimates depending on the two extremes for how deaths might be distributed. On one end, we assume all of the deaths took place among the symptomatic cases with no hospitalization category as shown in Panel B in Table 2 (with all of the other categories remaining the same). The total number of symptomatic cases with no hospitalization decreases from about 27.5 million to 27.3 million when the 147,000 deaths are removed. The overall valuation for symptomatic cases with no hospitalization drops from $\$ 899$ billion to $\$ 894$ billion. This translates into the overall non-fatal valuation decreasing by about $0.2 \%$.

On the other extreme, if we distribute all of the 147,000 deaths into the ICU total on mechanical ventilation group we are left with 85,000 victims on mechanical ventilation. This makes the overall non-fatal valuation decrease from about $\$ 2.2$ trillion to $\$ 1.8$ trillion (about a $20 \%$ decrease). The limited data from the CDC ( 2020f) on place of death suggests the fatalities likely crowd out more of the most severe non-fatal categories (and therefore, suggests the lower valuation total of about $\$ 1.8$ trillion would be closer to the real adjusted value), however, the exact amount is currently uncertain. ${ }^{6}$

\subsection{Applying estimates of value of a statistical injury (VSI)}

Viscusi and Gentry (2015) provide a range of the value of non-fatal statistical injury (VSI) estimates using the Census of Fatal Occupational Injuries (CFOI) data. They provide VSI estimates on both transportation and non-transportation injury types. Their transportation VSI estimates range in value between $\$ 83,000$ and $\$ 249,000$ (in 2019 dollars). As a comparison, their non-transportation VSI estimates range in value

\footnotetext{
5 The best data from the CDC (2020f) as viewed on July 27, 2020 show roughly 82,000 to 87,000 deaths took place within hospitals depending upon the categories used. This number increases by roughly another 33,000 when adding in nursing home and hospice totals. It is uncertain as to how the numbers would specifically breakdown across the various categories in Panel B in Table 2.

${ }^{6}$ For example, suppose we zero out losses from asymptomatic cases because such individuals may not even know they have COVID-19 and it might not impact their daily lives in any meaningful manner. Placing a zero value on the number of asymptomatic cases in Panel B in Table 2 would decrease the overall valuation from about $\$ 2.2$ trillion to $\$ 2.0$ trillion (about a $10 \%$ decrease), which is the midpoint of the interval just mentioned above.
} 
between $\$ 83,000$ and $\$ 95,000$ (in 2019 dollars). We apply now the Viscusi and Gentry (2015) non-transportation results because we believe they are more applicable to our COVID-19 non-fatal results. Their results show the median number of days away from work for non-fatal injuries range is 6-10 days depending upon industry and fatality type. About a quarter of the injuries required more than 31 days away from work. Injuries requiring less than two days away from work were about $15 \%$ of the observations.

In terms of COVID-19 cases, the CDC ( 2020g) guidelines generally recommend self-isolating for 10 days following symptom onset for mild to moderate cases. We equate such so-called mild and moderate cases with the asymptomatic and symptomatic with no hospitalization categories that we use. The 10-day self-isolation recommendation lines up on the higher end of the median number of days away from work values in Viscusi and Gentry (2015). For the more severe cases (hospitalizations) the CDC ( 2020g) recommends self-isolating for up to 20 days to mitigate transmission of the virus, although the numbers can be adjusted based upon advice from healthcare professionals. A related datum is the number of days the more severe COVID-19 cases spend in the hospital. The most recent CDC ( 2020b) estimates suggest the median (unweighted) number of hospitalization days among those not admitted to ICU is about 4 days and the median (unweighted) number of hospitalization days among those admitted to ICU is 12 days.

Although the combined number of hospitalization and self-isolation days for COVID-19 patients would be higher than the average number of days away from work values in Viscusi and Gentry (2015), it is unclear by how much given the information available in the main results. The higher-end results are likely to be substantially larger given the severity of some of the hospitalization cases (long-term lung damage, for example), however, we are reluctant to provide more specific values because that information is not available. As another possible estimate we note that if we use the lowest VSI value of $\$ 83,000$ for all non-fatal infections the total estimated loss from them is about $\$ 4$ trillion thus far.

\subsection{Takeaways from alternative calculations}

We have just provided a wide range of non-fatal adjustment valuations based upon the dreaded and uncertain aspects of COVID-19, age, income, and a factor related to fatality categorization. We find the largest potential decrease in the overall non-fatal valuations comes from the age adjustment ratio in Greenstone and Nigam (2020). The results suggest adjusting the non-fatal values by age might decrease the overall values by as much as $30 \%$. On the other extreme, adjusting the values by the dreaded and uncertainty factor associated with COVID-19 could dramatically increase the values by as much as $400 \%$ as suggested in Liu et al. (2005). The wide range of estimates suggests researchers should be cautious when applying imputed willingness to pay to avoid non-fatal pandemic outcome estimates for future COVID-19 analyses. At a minimum, we recommend using sensitivity analyses in future studies of the value of preventing non-fatal COVID-19 cases. 
Table 3 Forecast of Cases, Hospitalizations, ICU, and Ventilator Totals from July 28, 2020 through November 26, 2020

\begin{tabular}{llll}
\hline & Total & Severity Category & $\begin{array}{l}\text { Overall Valuation } \\
\text { (in billions) }\end{array}$ \\
\hline $\begin{array}{l}\text { Asymptomatic cases (no hospitalization) } \\
\text { Symptomatic cases (no hospitalization) }\end{array}$ & $30,017,078$ & $\$ 10,900$ & $\$ 327.2$ \\
Hospitalization total (minus those & $43,494,747$ & $\$ 32,700$ (Minor) & $\$ 1422.3$ \\
$\begin{array}{l}\text { in ICU and on ventilators) } \\
\text { ICU total (minus }\end{array}$ & $1,040,992$ & $\$ 512,300$ (Moderate) & $\$ 533.3$ \\
those on ventilators) & & & \\
ICU total & 122,470 & $\$ 1,144,500$ (Serious) & $\$ 140.2$ \\
$\begin{array}{l}\text { on mechanical ventilation } \\
\text { Total cases and overall valuation }\end{array}$ & $\mathbf{3 6 7 , 4 0 9}$ & $\mathbf{\$ 2 , 8 9 9 , 4 0 0 \text { (Severe) }}$ & $\mathbf{\$ 1 0 6 5 . 3}$ \\
$\begin{array}{l}\text { Non-Fatal Weighted Average Value } \\
\text { (Overall valuation/Total number of cases) }\end{array}$ & & & $\mathbf{\$ 3 4 8 8 . 2}$ \\
\hline
\end{tabular}

Notes: Forecast data taken from CDC ( 2020d) as viewed on July 27, 2020

Data available at: https://www.cdc.gov/coronavirus/2019-ncov/cases-updates/hospitalizations-forecasts.html Assumptions for categories taken from CDC (2020b; 2020c) as detailed in Table 1

\section{Issues for the near future}

\subsection{Infection predictions for July-November 2020}

The CDC provides forecasting data on the expected number of nationwide hospitalizations from July 28, 2020, through November 26, 2020, when the total number of hospitalizations is expected to be about 1.5 million (CDC 2020d). ${ }^{7}$ Table 3 shows how expected total hospitalizations break down across the various non-fatal categories when using the assumptions provided by the CDC (as shown in Table 1).

As shown in Table 3, during July-November 2020 total estimated cases will reach about 75 million. Non-fatal totals vary dramatically depending upon the category estimated. Asymptomatic cases are forecasted to be 30 million, and symptomatic cases with no hospitalization are expected to be about 43.5 million. Hospitalizations minus those in ICU and on ventilators are expected to total about 1 million, ICUs minus those on ventilators are forecast to be 122,000 , and ICUs on mechanical ventilation are expected to be about 367,000 during July-November 2020.

Overall valuations in Table 3 come from multiplying the non-fatal totals by their respective DOT severity values. The summation of asymptomatic cases ( $\$ 327$ billion), symptomatic cases with no hospitalization (\$1.4 trillion), hospitalizations not in ICU or on

\footnotetext{
${ }^{7}$ A variety of forecasting models are available for researchers to use including ones from Columbia University, the COVID-19 Simulator Consortium, the Institute for Health Metrics and Evaluation (IHME) and the Johns Hopkins University Infectious Disease Dynamics Lab (JHU) to name a few. We use the COVID-19 Simulator Consortium, which is a standard SEIR model that is based on assumptions about how levels of social distancing will change in the future and which assumes a $20 \%$ increase in mobility as each state reopens. Our results are robust to using a number of different forecasting models because many of them closely mirror each other in their final projections.
} 


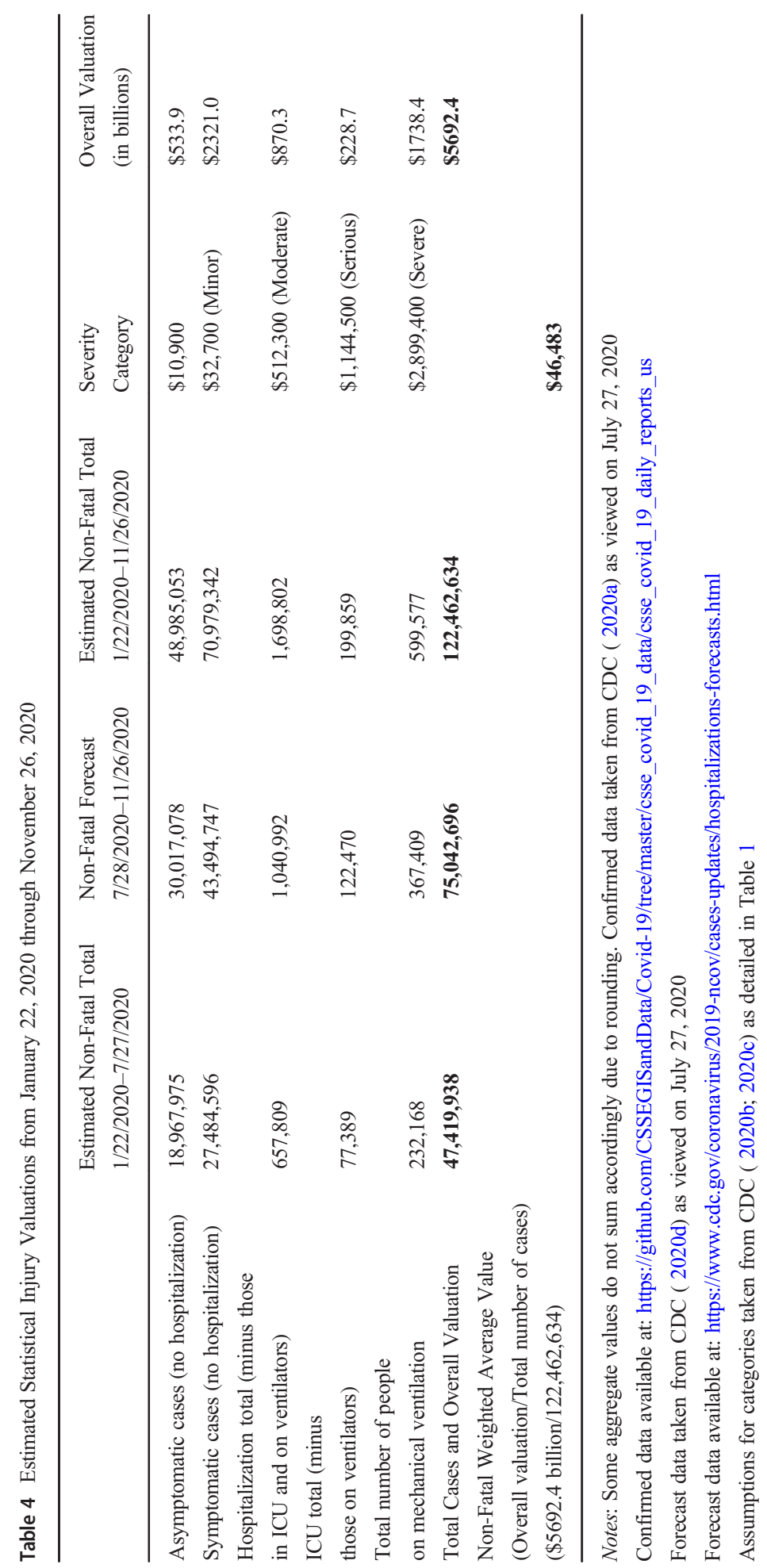


a ventilator ( $\$ 533$ billion), ICUs without being on a ventilator ( $\$ 140$ billion), and ICUs on a mechanical ventilator ( $\$ 1.1$ trillion) yields an expected overall non-fatal valuation of about $\$ 3.5$ trillion during the July 28, 2020, through November 26, 2020, time frame.

Table 4 combines the current estimates from January 22, 2020, through July 27, 2020, as shown in Table 2 with the forecasting data from July 28, 2020, through November 26, 2020, as detailed in Table 3. The total number of estimated cases up to July 27, 2020, is 47.4 million and cases forecasted for July 28, 2020, through November 26, 2020, are about 75 million. The combined number of estimated cases from January 22, 2020, through November 26, 2020, is then expected to be about 122 million. This represents almost $40 \%$ of the U.S. population given the recent census population estimate of about 328 million in 2019 (U.S. Census Bureau 2019).

Multiplying the estimated non-fatal totals by each category in Table 4 by their respective DOT severity values shows asymptomatic cases with an overall valuation of $\$ 534$ billion, symptomatic cases with no hospitalization at $\$ 2.3$ trillion, hospitalizations not in ICU or on a ventilator at $\$ 870$ billion, ICUs without being on a ventilator at $\$ 229$ billion, and ICUs on a mechanical ventilator at $\$ 1.7$ trillion. The total combined nonfatal valuation for the U.S. from January 22, 2020, through November 26, 2020, sums to almost $\$ 6$ trillion.

\subsection{Herd immunity}

As of July 27, 2020, we estimate roughly 47 million Americans have been infected with COVID-19. This corresponds to just over $14 \%$ of the current U.S. population (328 million) according to the most recent census figures (U.S. Census Bureau 2019). That said, the more long-term projections indicate the figures could rise to as high as 122 million Americans by the end of November - suggesting almost $40 \%$ of U.S. society may have been infected at some point by that time period. Although the pharmaceutical industry has focused enormous efforts on developing a safe vaccine, there is also the possibility of herd immunity developing naturally if the disease continues to spread at the rates we are seeing today.

There are two critical pieces needed for herd immunity to take hold in squelching a virus such as this one. The first is for individuals to have a low (or ideally zero) possibility of being re-infected. Although still somewhat uncertain, the emerging consensus among experts is that re-infection is unlikely for most individuals (Le Bert et al. 2020). If reinfection rates remain low the next requirement would be for society to reach the infection population thresholds needed for virus suppression.

Most experts estimate roughly $70 \%$ of the population would need to be infected by COVID-19 to reach herd immunity (Dowdy and D'Souza 2020; Mayo Clinic 2020). This means approximately 230 million Americans would need to be infected to achieve the required herd immunity thresholds as suggested by most medical experts. Infected totals in the United States would then have to rise by another 183 million people ( 230 million minus 47 million) to reach herd immunity. Looking at the long-term projections through the end of November (122 million cases) suggests we will still be short of the $70 \%$ threshold by about 108 million people (230 million minus 122 million).

Although the $70 \%$ threshold figure is currently the medical standard, some research suggests it could be much lower when one considers population heterogeneity (susceptibility by age). The most optimistic scenario appears to be recent research 
suggesting herd immunity thresholds for COVID-19 might be as low as 43\% (Britton et al. 2020). Using a $43 \%$ threshold would mean 141 million people would need to be infected before herd immunity takes hold - which is only 19 million people away from our long-term estimate (122 million) by the end of November 2020. Using the most optimistic assumptions in the literature, therefore, suggests herd immunity could take place as early as the first part of 2021.

All of the literature and case projections we provide indicate herd immunity will not take hold at any point in 2020. The best-case scenario indicates that the earliest herd immunity might take place would be in early 2021. However, using more standard assumptions suggests we are a long time away from reaching the normal population thresholds needed for herd immunity. As of late July 2020, we are roughly 180 million people away from herd immunity developing in society and by late November 2020 we will still be about 110 million people short of the normal thresholds needed. A scientific breakthrough on a safe vaccine could solve most of the issues, of course, but if one is not developed soon then our best estimates suggest herd immunity will not develop until at least late 2021 or 2022.

\section{Conclusion}

Our main points include that in the current pandemic economic policy decisions may be at least as important to society as medical policy decisions. One need only examine the NBER Working paper series and the new journal, Covid Economics, to become convinced. Most of the literature to date has focused on the most serious of all medical outcomes, death. (For background see Viscusi 2018 and Kniesner \& Viscusi 2019) We contend that this has led policymakers to largely ignore the importance of non-fatal cases of infection and their implied economic consequences. At a minimum this would involve changing the equation for the net economic benefit of interdicting the spread of COVID-19 to add the gains from preventing the various types of non-fatal infection cases net of any additional attendant prevention costs. Put simply, more of the details of the importance of preventing the spread of infection and the costs of doing so need to be fleshed out in public decisions.

Although it is the case that so-called herd immunity achieved by people who get non-fatal infections is of value in possibly stemming the pandemic in the absence of an effective vaccine, and may be as low as about $40 \%$ (Britton et al. 2020), and that people may suffer emotionally from a lack of varied human contact during a lockdown, this needs to be balanced against the losses people suffer from non-fatal cases of COVID19. We have attempted to demonstrate here that non-fatal infections from COVID-19 are just as important economically in the aggregate as the losses from fatal infections to the affected persons because of the much greater numbers involved, the incompletely appreciated seriousness of the non-fatal infections and the dread people suffer from not knowing whether their infection will become fatal ultimately. The near future is likely to bring as many as 75 million new non-fatal COVID-19 cases by the end of 2020 with little in the way of policy other than protective masks and social distancing. Our calculations imply that policymakers must enlighten the public that non-fatal infections can be prevented and are as economically serious in the aggregate as ultimately fatal infections because of the large numbers of cases involved. 
Acknowledgments Views expressed here do not reflect official policy or position of the U.S. Department of Defense or the U.S. Federal government. We thank James Hammitt, Lisa Robinson, and W. Kip Viscusi for helpful comments.

\section{References}

Adler, M. D. (2020). What should we spend to save lives in a pandemic? A critique of the value of statistical life. Covid Economics, 33(June 30), 1-45.

Britton, T., Ball, F., \& Trapman, P. (2020). A mathematical model reveals the influence of population heterogeneity on herd immunity to SARS-CoV-2. Science, June 23.

Centers for Disease Control and Prevention. (2020a). COVID-19 daily reports. Available at: https://github. com/CSSEGISandData/COVID-19/tree/master/csse_COVID_19_data/csse_COVID_19_daily_reports_ us.

Centers for Disease Control and Prevention. (2020b). COVID-19 pandemic planning scenarios. Available at: https://www.cdc.gov/coronavirus/2019-ncov/hcp/planning-scenarios-h.pdf.

Centers for Disease Control and Prevention. (2020c). Cases, data, and surveillance. Available at: https://www. cdc.gov/coronavirus/2019-ncov/cases-updates/commercial-lab-surveys.html\#interpreting-serologyresults.

Centers for Disease Control and Prevention. (2020d). Hospitalization forecasts. Available at: https://www.cdc. gov/coronavirus/2019-ncov/cases-updates/hospitalizations-forecasts.html.

Centers for Disease Control and Prevention. (2020e). Morbidity and Mortality Weekly Report (January $22-$ May 30, 2020). Available at: https:/www.cdc.gov/mmwr/volumes/69/wr/pdfs/mm6924e2-H.pdf.

Centers for Disease Control and Prevention. (2020f). Provisional COVID-19 death counts by place of death and state. Available at: https://data.cdc.gov/NCHS/Provisional-COVID-19-Death-Counts-by-Place-ofDeat/uggs-hy5q.

Centers for Disease Control and Prevention. (2020g). Duration of isolation and precautions for adults with COVID-19. Available at: https://www.cdc.gov/coronavirus/2019-ncov/hcp/duration-isolation.html.

Centers for Medicare and Medicaid Services. (2020). Preliminary Medicare COVID-19 data snapshot. Available at: https://www.cms.gov/files/document/medicare-COVID-19-data-snapshot-fact-sheet.pdf.

Chilton, S., Jones-Lee, M., Kiraly, F., Metcalf, H., \& Pang, W. (2006). Dread risks. Journal of Risk and Uncertainty, 33(3),165-182.

Dowdy, D., \& D’Souza, G. (2020). Early herd immunity against COVID-19: A dangerous misconception. Johns Hopkins University and Medicine Coronavirus Resource Center. Available at: https:/coronavirus. jhu.edu/from-our-experts/early-herd-immunity-against-COVID-19-a-dangerous-misconception.

Gayer, T., Hamilton, J. T., \& Viscusi, W. K. (2000). Private values of risk tradeoffs at superfund sites: housing market evidence on learning about risk. Review of Economics and Statistics, 82(3), 439-451.

Greenstone, M., \& Nigam, V. (2020). Does social distancing matter? Covid Economics, 7, 1-22.

Gyrd-Hansen, D., Halvorsen, P. A., \& Kristiansen, I. S. (2008). Willingness to pay for a statistical life in the times of a pandemic. Health Economics, 17(1), 55-66.

Hall, R.E., Jones, C.I., \& Klenow, P.J. (2020). Trading off consumption and COVID-19 deaths. National Bureau of Economic Research (no. w27340).

Hammitt, J.K. (2020). Valuing mortality risk in the time of COVID-19. Journal of Risk and Uncertainty, 61(2). (in press).

Hammitt, J. K., \& Liu, J. T. (2004). Effects of disease type and latency on the value of mortality risk. Journal of Risk and Uncertainty, 28(1), 73-95.

Hammitt, J. K., \& Robinson, L. A. (2011). The income elasticity of the value per statistical life: Transferring estimates between high and low income populations. Journal of Benefit-Cost Analysis, 2(1), 1-29.

Interlandi, J. (2020). Why we're losing the battle with COVID-19. New York Times Magazine, July 19.

Jones-Lee, M. W., \& Loomes, G. (1995). Scale and context effects in the valuation of transport safety. Journal of Risk and Uncertainty, 11(3), 183-203.

Kniesner, T. J., \& Viscusi, W. K. (2019). Value of a statistical life. Oxford Research Encyclopedia, Economics and Finance. https://doi.org/10.1093/acrefore/9780190625979.013.138.

Kniesner, T. J., Viscusi, W. K., \& Ziliak, J. P. (2010). Policy relevant heterogeneity in the value of statistical life: New evidence from panel data quantile regressions. Journal of Risk and Uncertainty, 40(1), 15-31. 
Le Bert, N., Tan, A. T., Kunasegaran, K., Tham, C. Y., Hafezi, M., Chia, A., Chng, M. H. Y., Lin, M., Tan, N., Linster, M., \& Chia, W. N. (2020). SARS-CoV-2-specific T-cell immunity in cases of COVID-19 and SARS, and uninfected controls. Nature, 7, 1-10.

Lindhjem, H., Navrud, S., Braathen, N. A., \& Biausque, V. (2011). Valuing mortality risk reductions from environmental, transport, and health policies: A global meta-analysis of stated preference studies. Risk Analysis, 31(9), 1381-1407.

Liu, J. T., Hammitt, J. K., Wang, J. D., \& Tsou, M. W. (2005). Valuation of the risk of SARS in Taiwan. Health Economics, 14(1), 83-91.

Manski, C. (2020a). Adaptive diversification of COVID-19 policy. VoxEU, June 12. https://voxeu.org/article/ adaptive-diversification-COVID-19-policy.

Manski, C. (2020b). COVID-19 policy must take all impacts into account. Scientific American, March 28. https://blogs.scientificamerican.com/observations/COVID-19-policy-must-take-all-impacts-into-account.

Masterman, C. J., \& Viscusi, W. K. (2018). The income elasticity of global values of a statistical life: Stated preference evidence. Journal of Benefit-Cost Analysis, 9(3), 407-434.

Mayo Clinic. (2020). Herd immunity and COVID-19 (coronavirus): What you need to know. Available at: https://www.mayoclinic.org/diseases-conditions/coronavirus/in-depth/herd-immunity-and-coronavirus/ art-20486808.

McDonald, R. L., Chilton, S. M., Jones-Lee, M. W., \& Metcalf, H. R. (2016). Dread and latency impacts on a VSL for cancer risk reductions. Journal of Risk and Uncertainty, 52(2), 137-161.

Miller, H. J. (2020). COVID's harrowing complications. Wall Street Journal, July 20.

New York Times. (2020). Actual coronavirus infections vastly undercounted, C.D.C. data shows. Available at: https://www.nytimes.com/2020/06/27/health/coronavirus-antibodies-asymptomatic.html.

Oran, D. P., \& Topol, E. J. (2020). Prevalence of asymptomatic SARS-CoV-2 infection: A narrative review. Annals of Internal Medicine. Available at: https://www.acpjournals.org/doi/full/10.7326/M20-3012, 173(5), 362-367.

Plaschke, B. (2020). He took COVID-19 seriously, then it hit him personally. Los Angeles Times, 13, B6.

Riddel, M., \& Shaw, W. D. (2006). A theoretically-consistent empirical model of non-expected utility: An application to nuclear-waste transport. Journal of Risk and Uncertainty, 32(2), 131-150.

Robinson, L. A., Sullivan, R. S., \& Shogren, J. F. (2020). Do the benefits of COVID-19 policies exceed the costs? Exploring uncertainties in the age-VSL relationship. Risk Analysis. https://doi.org/10.1111/risa. 13561.

Rowell, A. (2020). COVID VSLs and the undervaluation of pandemic risk. The Regulatory Review, Penn Program on Regulation, August 17.

Spicer, R.S., \& Miller, T.R. (2010). Final report to the National Highway Traffic Safety Administration. Available at: https:/www.transportation.gov/sites/dot.gov/files/docs/Spicer\%20and\%20Miller\% 202010\%20qaly_injury_revision_pdf_final_report_02-05-10.pdf.

Thunström, L., Newbold, S., Finnoff, D., Ashworth, M., \& Shogren, J. F. (2020). The benefits and costs of flattening the curve for COVID-19. Journal of Benefit-Cost Analysis, 11(2), 179-195.

U.S. Census Bureau. (2019). National Population by Characteristics: 2010-2019. Available at: https://www. census.gov/data/datasets/time-series/demo/popest/2010s-national-detail.html.

U.S. Department of Health and Human Services. (2016). Guidelines for regulatory impact analysis, Washington, DC. https://aspe.hhs.gov/pdf-report/guidelines-regulatory-impact-analysis.

U.S. Department of Transportation. (2016). Revised Department Guidance on Valuation of a Statistical Life in Economic Analysis. Available at: https:/www.transportation.gov/sites/dot.gov/files/docs/2016\% 20Revised $\% 20$ Value $\% 20$ of $\% 20$ a\%20Statistical\%20Life\%20Guidance.pdf.

U.S. Environmental Protection Agency. (2009). Second Section 812 Prospective Analysis. Available at: https://www.epa.gov/sites/production/files/2015-07/documents/dec2009_benefitanal_ch1and2.pdf.

Viscusi, W. K. (2018). Pricing lives: Guideposts for a safer society. Princeton: Princeton University Press.

Viscusi, W. K. (2019). Utility functions for mild and severe health risks. Journal of Risk and Uncertainty, 58(2-3), 143-166.

Viscusi, W.K. (2020). Pricing the global health risks of the COVID-19 pandemic. Journal of Risk and Uncertainty, 61(2). (in press).

Viscusi, W. K., \& Aldy, J. E. (2003). The value of a statistical life: A critical review of market estimates throughout the world. Journal of Risk and Uncertainty, 27(1), 5-76.

Viscusi, W. K., \& Gentry, E. P. (2015). The value of a statistical life for transportation regulations: A test of the benefits transfer methodology. Journal of Risk and Uncertainty, 51(1), 53-77.

Viscusi, W. K., Huber, J., \& Bell, J. (2014). Assessing whether there is a cancer premium for the value of a statistical life. Health Economics, 23(4), 384-396. 
Viscusi, W. K., \& Masterman, C. (2017a). Anchoring biases in international estimates of the value of a statistical life. Journal of Risk and Uncertainty, 54(2), 103-128.

Viscusi, W. K., \& Masterman, C. J. (2017b). Income elasticities and global values of a statistical life. Journal of Benefit-Cost Analysis, 8(2), 226-250.

Washington Post. (2020). CDC chief says coronavirus cases may be 10 times higher than reported. Available at: https://www.washingtonpost.com/health/2020/06/25/coronavirus-cases-10-times-larger/.

Weinberger, D.M., Chen, J., Cohen, T., Crawford, F.W., Mostashari, F., Olson, D., Pitzer, V.E., Reich, N.G., Russi, M., Simonsen, L., \& Watkins, A. (2020). Estimation of excess deaths associated with the COVID19 pandemic in the United States, March to May 2020. JAMA Internal Medicine, July 1.

Publisher's note Springer Nature remains neutral with regard to jurisdictional claims in published maps and institutional affiliations. 